This document is the unedited Author's version of a Submitted Work that was subsequently accepted for publication in Accounts of Chemical Research, copyright (c) American Chemical Society after peer review. To access the final edited and published work see:

https://dx.doi.org/10.1021/acs. accounts. 8b00241. 


\section{Unraveling the Operational Mechanisms of \\ Chemically Propelled Motors with Micropumps}

Maria Jose Esplandiu ${ }^{\dagger, *}$ Kuan Zhang ${ }^{\dagger}$, Jordi Fraxedas ${ }^{\dagger}$, Borja Sepulvedat and David Reguera $\$ \$$

$\dagger$ Catalan Institute of Nanoscience and Nanotechnology (ICN2), CSIC and BIST, Campus UAB, Bellaterra, 08193 Barcelona, Spain

†Departament de Física de la Matèria Condensada, Universitat de Barcelona, C/Martí i Franquès 1, 08028 Barcelona, Spain.

§Universitat de Barcelona Institute of Complex Systems (UBICS), C/Martí i Franquès 1, 08028 Barcelona, Spain.

CONSPECTUS. The development of effective autonomous micro/nanomotors relies on controlling fluid motion at interfaces. One of the main challenges in the engineering of such artificial machines is the quest for efficient mechanisms to power them without using external driving forces. In the last decade, there has been an important upraise of man-made micro/nanomotors fueled by self-generated physicochemical gradients. Impressive proofs of concept of multitasking machines have been reported demonstrating their capabilities for a plethora of applications. While the progress toward applications is promising, there are still open questions on fundamental physicochemical aspects behind the mechanical actuation, which 
require more experimental and theoretical efforts. These efforts are not merely academic, but will open the door for an efficient and practical implementation of such promising devices.

In this account we focus on chemically driven motors whose motion is the result of a complex interplay of chemical reactions and (electro)hydrodynamic phenomena. A reliable study of these processes is rather difficult with mobile objects like swimming motors. However, pumps, which are the immobilized motor counterparts, emerge as simple manufacturing and well-defined platforms for a better experimental probing of the mechanisms and key parameters controlling the actuation.

Here we review some recent studies using a new methodology which has turned out to be very helpful to characterize micropump chemomechanics. The aim was to identify the redox role of the motor components, to map the chemical reaction and to quantify the relevant electrokinetic parameters (e.g., electric field and fluid flow). This was achieved by monitoring the velocity of differently charged tracers and by fluorescence imaging of the chemical species involved in the chemical reaction, e.g., proton gradients. We applied these techniques to different systems of interest. First, we probed bimetallic pumps as counterparts of the pioneering bimetallic swimmers. We corroborated that fluid motion was due to a self-generated electro-osmotic mechanism driven by the redox decomposition of $\mathrm{H}_{2} \mathrm{O}_{2}$. In addition, we analysed by simulations the key parameters that yield an optimized operation. Moreover, we accomplished a better assessment of the importance of surface chemistry on the metal electrochemical response, highlighting its relevance in controlling the redox role of the metals and motion direction.

Second, we focused on metallic/semiconductor micropumps to analyze light-controlled motion mechanisms through photoelectrochemical decomposition of fuels. These pumps were driven by 
visible light and could operate using just water as fuel. In these systems, we found a very interesting competition between two different mechanisms for fluid propulsion, namely lightactivated electro-osmosis and light-insensitive diffusio-osmosis, stemming from different chemical pathways in the fuel decomposition. In this case, surface roughness becomes a pivotal parameter to enhance/depress one mechanism over the other.

These examples demonstrate that pumps are practical platforms to inquire operating mechanisms and to quantify their performance. Additionally, they are suitable systems to test novel fuels or motor materials. This knowledge is extensible to swimmers providing not only fundamental understanding of their locomotion mechanisms but also useful clues for their design and optimization.

\section{INTRODUCTION}

The engineering of self-propelled micro/nanomachines with the capability to emulate the complex functionalities of biological systems has become a research line of growing interest since the pioneering studies at the beginning of this millennium ${ }^{1,2}$. These micro/nanomachines, which generate and harness local physico-chemical gradients to drive their own motion, show potential to become autonomous multi-tasking micro/nanorobots as one of the long-awaited challenges of nanotechnology $y^{3-6}$. The number of studies highlighting different potential applications of micro/nanoswimmers is remarkable. It has been demonstrated that they can capture, transport and deliver loads ${ }^{7}$, target specific environments ${ }^{8}$, image ${ }^{9}$, sense ${ }^{10}$ and neutralize/degrade (bio)chemicals ${ }^{11-13}$ or induce collective behaviors ${ }^{14}$ (Fig.1). Similarly, although to a lesser extent, micropumps sharing the same working principles as microswimmers, are envisioned as smart devices for manipulating and guiding fluid flows. Proof of concept 
experiments have placed micropumps as promising platforms for providing controlled mass release, mass transport/ accumulation/clearance, for material patterning at precise locations or for sensing applications by triggering fluid motion when adding certain chemical analytes (Fig.2) ${ }^{15-}$ 17. Although demonstrations in real-life applications are still missing, it is expected that swimming motors and pumps will have an important impact on microfluidics, lab-on-a chip devices, nanomedicine and environmental remediation.

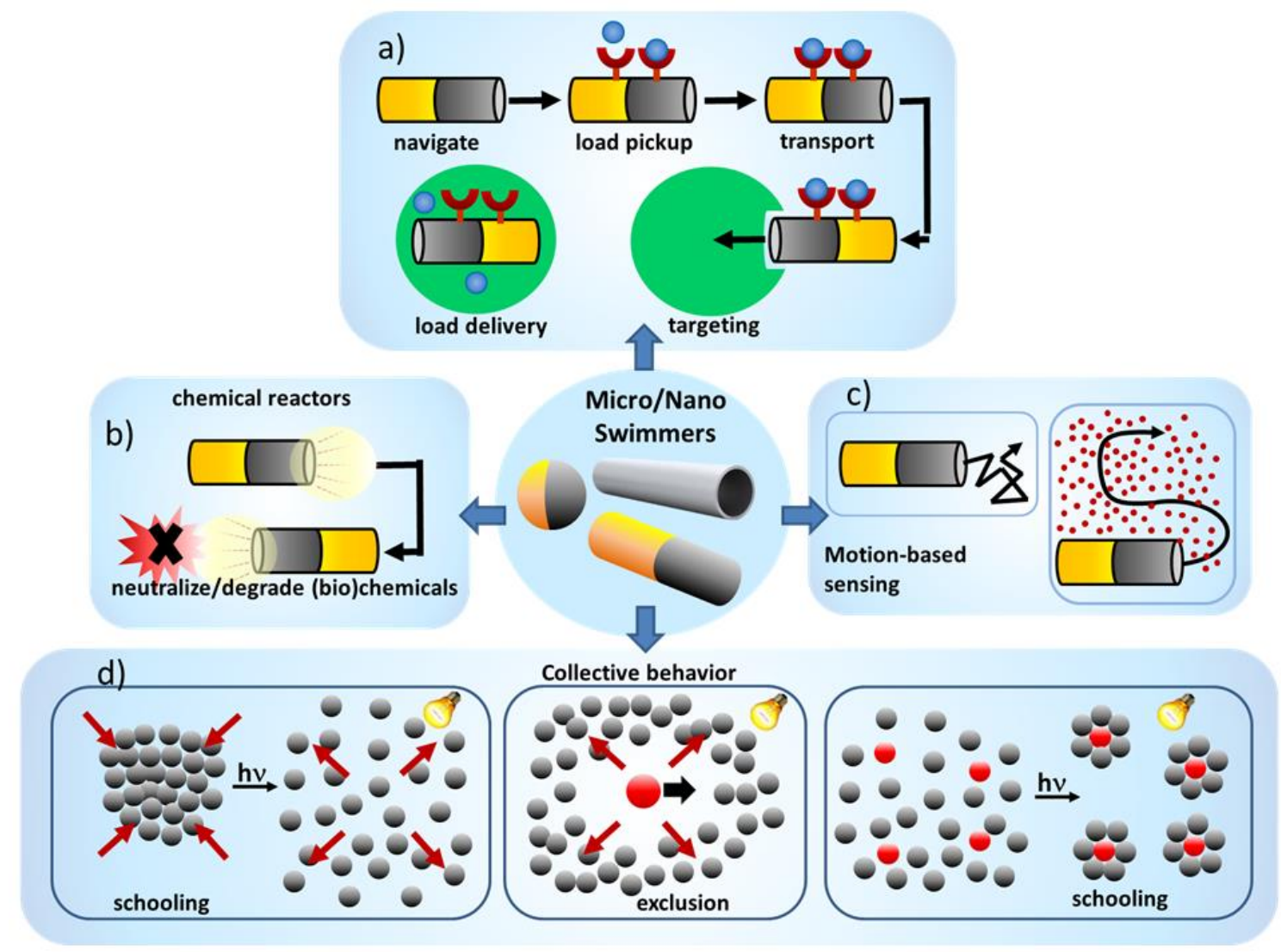

Figure 1. Different tasks performed by micro/nanoswimmers: a) cargo load, transport and delivery at targeting places; b) mobile chemical reactors for degrading/neutralizing chemical species; c) sensors which can be activated in the presence of certain chemical species; d) particle 
recruitment or exclusion as a consequence of the interaction of their self-generated chemical gradients.

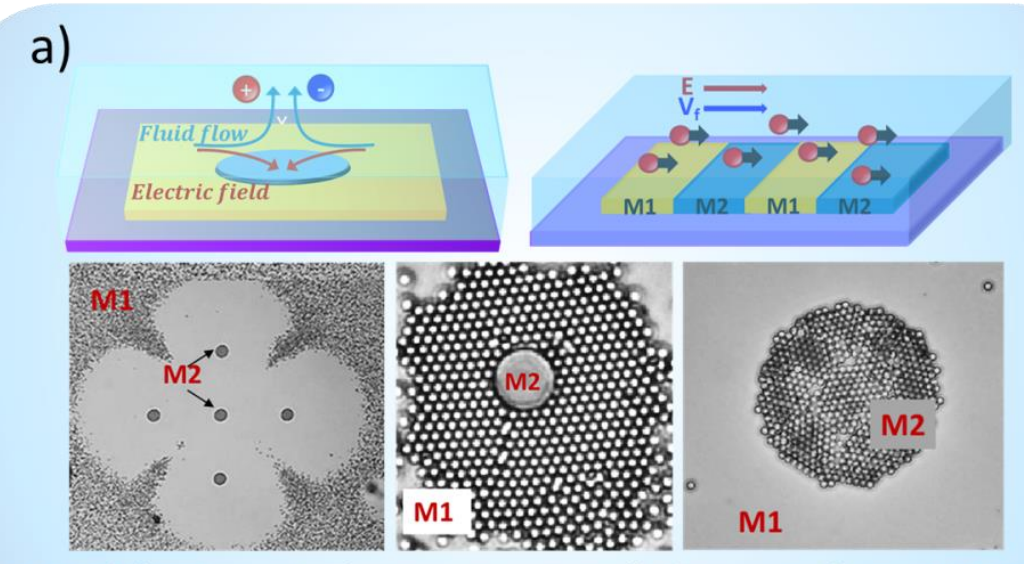

Material transport, clearance, accumulation or surface patterning

b)

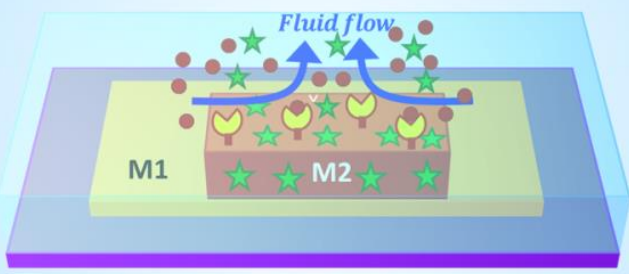

Local material release

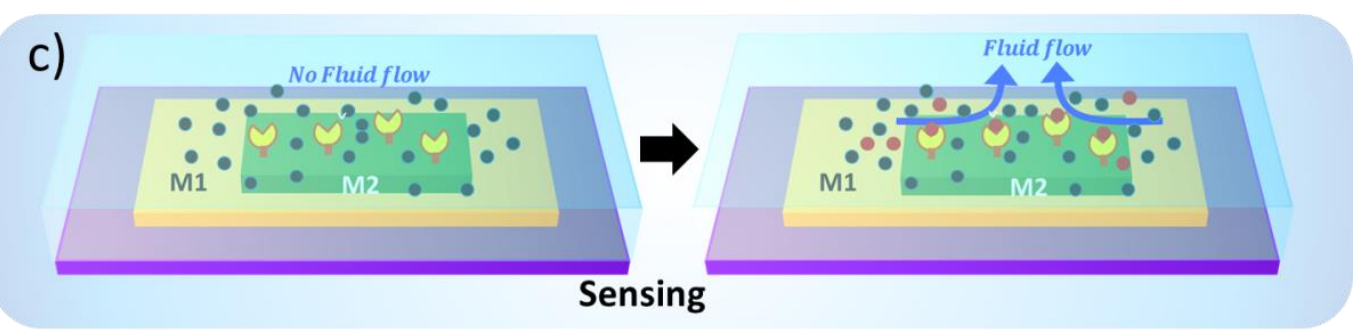

Figure 2. Different tasks performed by micropumps: a) mass transport, clearance, accumulation or self-assembly at specific locations; b) delivery of trapped materials with fluid flow activation; c) pumps modified with receptors acting as fluid motion-based sensors (e.g. fluid pumping can be triggered when the interaction of a receptor with a ligand generates a chemical gradient). 
Although the proofs of concept have been significant, the progress on the comprehension of the physicochemical fundamentals behind the self-generated actuation of swimmers/pumps has been more moderate. In many cases, the precise motion mechanism is still not unambiguously identified, and the key physicochemical parameters are not well-characterized. A complete and deep understanding of such issues would help to improve the control levels for applications and to better assess perspectives and challenges.

Different self-propulsion mechanisms can be operating in these machines depending on their composition, shape, size, fuel nature and concentration. The main mechanisms triggered by chemical reactions are summarized in Fig.3. 


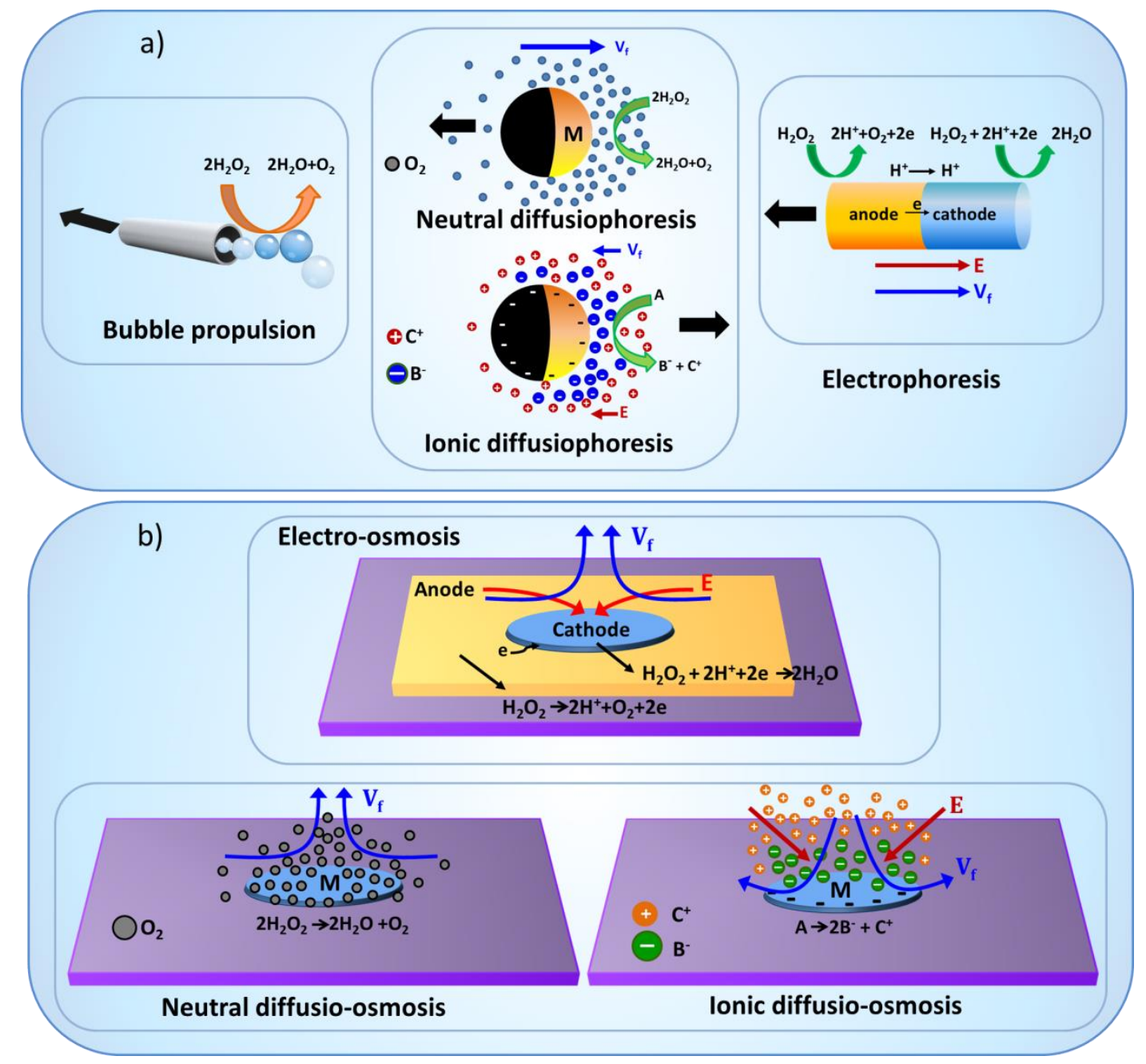

Figure 3. Schematics of different chemically propelled mechanisms in a) swimmers and b) pumps.

Bubble propulsion of swimmers relies on gas bubble formation from the chemical decomposition of a fuel at the motor catalyst interface ${ }^{5}$. Typical examples are Janus microparticles and tubular motors fueled by the decomposition of $\mathrm{H}_{2} \mathrm{O}_{2}$ into water and oxygen. A recoil force is generated due to a momentum change upon oxygen bubble detachment from the catalyst surface.

The alternatives to bubble propulsion are phoretic mechanisms driven by physicochemical gradients. In particular, at isothermal conditions, three different mechanisms can take place: 
neutral diffusiophoresis (concentration gradient of neutral solutes), ionic diffusiophoresis (gradient of salt or ionic species) and electrophoresis (electric potential gradient) ${ }^{18}$. Typically, these mechanisms are triggered by externally imposed gradients. However, in the case of swimmers/pumps, the goal is that these gradients are generated by the own motor from chemical reactions $^{19}$.

In the case of neutral diffusiophoresis, the motion direction and speed depend on the liquid interaction with the motor surface. In the simplest case of excluded volume interactions, a fluid flow directed toward higher solute concentration is induced, leading to motor motion in the opposite direction ${ }^{18}$. For ionic diffusiophoresis, the chemomechanical actuation depends on both chemiphoretic and electrophoretic contributions. The chemiphoretic contribution, due to pressure unbalance at the double layer, always induces liquid flow toward lower ion concentration. The electrophoretic effect arises as a consequence of having different ion diffusion coefficients in the solution which build up an electric field. Such electric field triggers a fluid flow at the motor interface whose direction depends on the electric field sign and the motor surface charge. The net fluid flow and motor direction are finally determined by the balance of these two contributions ${ }^{18}$.

Swimmer propulsion by pure electrophoresis can be generated when half redox reactions are induced separately at different places of the motor ${ }^{19}$. The production and consumption of ionic species at different motor locations induce an electric field which triggers the fluid flow at the motor interface. This mechanism is discussed in more detail later.

The same mechanisms operate in micropumps, under the name of electro- and diffusioosmosis $^{19-21}$. A pump can be considered as an immobilized motor, with the fluid flowing relative to its interface triggered by electric potential or concentration gradients. 
In contrast to traditional phoretic mechanisms driven by externally imposed gradients, selfgenerated phoretic motion involves an additional parameter: the chemical reaction. The chemical reaction is now coupled to the equations governing mass/charge transport, electric field and fluid flow which make the overall understanding of motion more complex ${ }^{22,23}$. Moreover, the analysis and quantification of a moving object such as a swimming motor makes its study rather challenging. As a consequence, there are still many unsolved questions regarding even which is the dominant mechanism driving these systems. A significant example is the still ongoing debate on whether Janus swimmers, made of a half insulator and half metal catalyst, move by electro or diffusiophoresis ${ }^{24,25}$.

In this context, catalytic pumps emerge as model systems for better probing the mechanism controlling phoretic motion. Effective experimental methods can be implemented more easily to spatially quantify the key parameters involved in such coupled processes (i.e., chemical rates and gradients, electric fields, fluid flow, zeta potential, etc.). Moreover, catalytic pumps have the advantage of being better defined and controlled from the point of view of component shape, size, surface chemical composition and roughness, which are important aspects affecting the catalytic motion and not so easy to control in swimmers.

Accordingly, in this review we recapitulate on a combined set of techniques that we have implemented to study chemically propelled micropumps. These techniques have turned out to be very useful for extracting physicochemical parameters and to achieve a more complete characterization of the mechanisms driving fluid motion. We have used these tools to unravel bimetallic pump actuation by mapping chemical gradients and relevant electrokinetic parameters, contrasting experiments with numerical simulations ${ }^{26,27}$. These studies have also revealed the importance of surface chemistry in controlling the involved reactions. We have also explored 
metal/semiconductor pumps to get a better understanding of light-driven actuation ${ }^{28}$. We will show that, in this case, the chemomechanical actuation also features competing mechanisms that dictate the final motion. Surface roughness becomes a pivotal parameter that determines the prevalence of one mechanism over the other ${ }^{29}$.

These studies with pumps are very relevant for their swimmer counterparts, shedding light on their motion mechanisms and providing useful clues for the design and optimization of phoretic systems.

\section{BIMETALLIC PUMPS}

\subsection{Chemical reaction mapping and quantification of electrokinetic parameters}

Research efforts were first devoted to characterize Au/Pt micropumps in $\mathrm{H}_{2} \mathrm{O}_{2}$, since they represent the analogs of the pioneering $\mathrm{Au} / \mathrm{Pt}$ swimmers $^{26}$. Many details were unknown on the mechanism itself, the electrochemical reaction production, and electric field generation. We applied a set of experimental techniques complemented with numerical simulations to provide a better scenario of the chemomechanical actuation ${ }^{26,27}$.

Micropumps were fabricated by patterning 30-50 $\mu \mathrm{m}$ diameter platinum discs on gold surfaces using electron-beam lithography or through physical masks (stencils) followed by electron-beam evaporation and oxygen plasma treatment. The micropumps were then immersed in $\mathrm{H}_{2} \mathrm{O}_{2}$ to trigger the fluid motion. In order to probe the mechanism and quantify the relevant electrohydrodynamic parameters, we optically monitored the motion of differently charged particles. Specifically, we used positive particles (with a zeta potential $\xi_{p^{+}}=46 \mathrm{mV}$ ), negative particles $\left(\xi_{p}=-83 \mathrm{mV}\right)$ and quasi-neutral particles $\left(\xi_{p 0}=-12 \mathrm{mV}\right)$. 

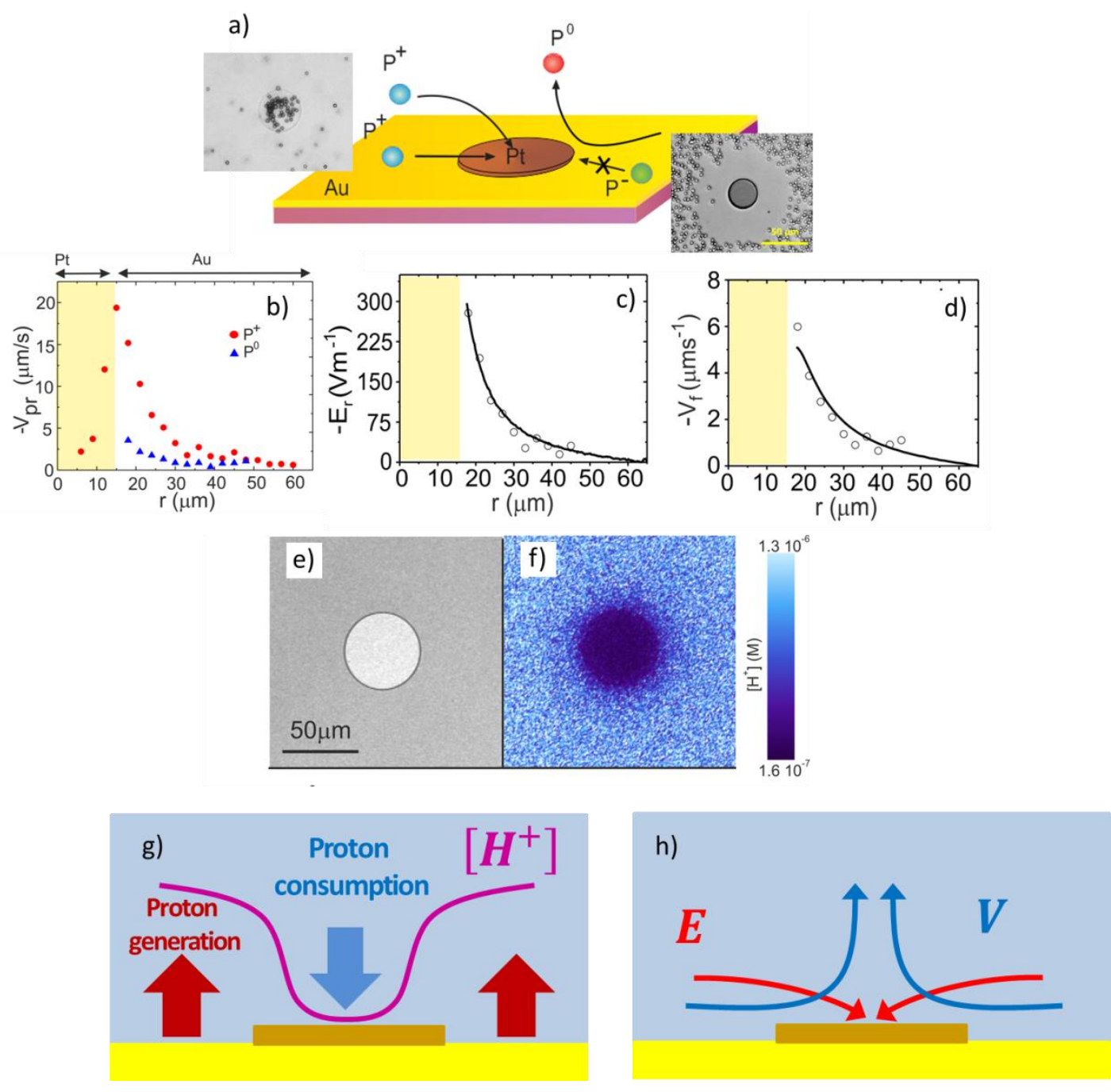

Figure 4. a) Scheme depicting the behavior of differently charged tracers together with optical images of the pumps interacting with positive and negative tracers in $1 \% \mathrm{H}_{2} \mathrm{O}_{2}$. b) Radial velocity of positive $\left(\mathrm{p}^{+}\right)$and quasi-neutral $\left(\mathrm{p}^{0}\right)$ particles as a function of the radial coordinate. The plot origin corresponds to the Pt disc center that spans an area represented by the yellow region. Experimental (circles) and simulation (solid lines) results of the radial electric field (c) and fluid velocity (d) showing an excellent agreement. Optical image of the Pt/Au pump (e) together with a proton mapping extracted from fluorescence measurements with $\mathrm{pH}$ indicators (f). Schematics representing proton production and consumption (g) and electric field and fluid 
flow direction at the pump (h). Figures 4 a-f reprinted with permission from ref. 26. Copyright 2013 by The American Physical Society.

The motion analysis of differently-charged tracers represents a powerful strategy to extract the strength and direction of the self-generated electric field and fluid flow. This was accomplished by considering that the radial velocity $\left(V_{r p}\right)$ of the tracer particles has two contributions: one coming from their charge (electrophoretic velocity, $V_{e f}$ ) and the other arisen from the fluid flow $\left(V_{r f}\right)$ :

$$
V_{r p}=V_{e f}+V_{r f}=\frac{\varepsilon \xi_{p} E_{r}}{\eta}+V_{r f}
$$

where $\varepsilon$ is the fluid permittivity, $\xi_{p}$ the particle zeta potential, $E_{r}$ the electric field radial component, and $\eta$ the fluid viscosity. Positive and negative particles are more sensitive to the electric field whereas quasi-neutral particles interact weakly with it hence being especially useful for tracking the fluid flow. Figure 4a summarizes the different motion types observed. Positive tracers moved toward the Pt disc, whereas negative particles got expelled forming a repulsion area around the Pt edge. Such behavior indicated the presence of an electric field pointing toward the Pt disc. Quasi-neutral particles moved toward the disc but then drifted upwards in the direction normal to the disc tracing the liquid's flow. Figures $4 \mathrm{c}$ and $\mathrm{d}$ show the quantification of the radial strength of the electric field and fluid flow extracted from the velocities of positive $\left(\mathrm{p}^{+}\right)$and quasi-neutral $\left(\mathrm{p}^{0}\right)$ particles (Fig. $\left.4 \mathrm{~b}\right)$ using eq. 1,

$$
\begin{aligned}
& E_{r}(r)=\frac{\eta\left(v_{r}^{p+}(r)-V_{r}^{p 0}(r)\right)}{\varepsilon\left(\xi_{p+}-\xi_{p 0}\right)} \\
& V_{f}(r)=\frac{\xi_{p+} v_{r}^{p 0}(r)-\xi_{p 0} v_{r}^{p+}(r)}{\xi_{p+}-\xi_{p 0}} .
\end{aligned}
$$


Both the radial fluid velocity and electric field increase as the disc center is approached. With the calculated values of $E_{r}(r)$ and $V_{f}(r)$ the substrate zeta potential $\left(\xi_{w}\right)$ was estimated from the standard expression of the electro-osmotic velocity:

$$
V_{f}(r)=-\frac{\varepsilon \xi_{w} E_{r(r)}}{\eta}
$$

yielding a reasonable value $(-33 \mathrm{mV})$ expected for noble metals ${ }^{21,26}$.

The electric field and fluid flow direction, pointing from Au toward Pt, were surprisingly opposite to the ones found on their $\mathrm{Au} / \mathrm{Pt}$ swimmers counterparts. In the case of swimmers $\mathrm{Pt}$ acted as the anode for $\mathrm{H}_{2} \mathrm{O}_{2}$ decomposition, with the consequent proton production, whereas $\mathrm{Au}$ acted as the cathode, with proton consumption, inducing an electric field from $\mathrm{Pt}$ to $\mathrm{Au}^{20}$. In order to probe the electrochemical reaction in our bimetallic pumps, we mapped the proton distribution with fluorescent $\mathrm{pH}$ indicators using ratiometric confocal fluorescence microscopy ${ }^{26}$. It was found that the proton concentration decreases as approaching the Pt disc, changing by almost one order of magnitude along the radial direction. These measurements proved that in our case protons are produced at $\mathrm{Au}$ and move towards the Pt disc where they are consumed. This confirms the roles of $\mathrm{Au}$ and $\mathrm{Pt}$ as anode and cathode, respectively, in our pumps. The discrepancy in the direction of the redox reaction, and electric and velocity fields in the $\mathrm{Au} / \mathrm{Pt}$ pumps as compared to the swimmers, highlights the importance of the metal electrode surface chemistry, as explained in the next section ${ }^{26}$.

The experimental results were confirmed and reproduced by numerical simulations solving, in a coupled way, the governing equations of the electrochemical reaction, mass and charge transport, electric field and fluid flow ${ }^{26}$. The solid lines in Fig. $4 \mathrm{c}$ and d show an excellent agreement 
between experimental data and simulations for the spatial dependence of the electric field and fluid flow. The simulations also demonstrated that the electric field extends above the double layer in the region of electroneutrality and confirmed that it is originated by a proton current from anode to cathode rather than by any lateral charge asymmetry inside the double layer ${ }^{27}$. In addition, simulations were used to assess how micropump performance depends on parameters such as zeta potential, ionic strength or geometry, giving important clues for achieving an optimal motor operation ${ }^{27}$.

Altogether, our studies provided a complete picture of the operational mechanism of catalytic micropumps. The pump works by electro-osmosis driven by an electric field generated by $\mathrm{H}_{2} \mathrm{O}_{2}$ oxidation and reduction at $\mathrm{Au}$ and $\mathrm{Pt}$, respectively. The redox reaction produces protons at the anode that are consumed at the cathode, building up a proton gradient that induces a net current. The proton current from anode to cathode generates an electric field triggering a fluid flow by electro-osmosis ${ }^{26}$. For pumps with negative surface potential, as in our case, the flow occurs in the same direction of the electric field.

\subsection{Surface chemistry}

Another important conclusion of those studies is the crucial role of surface chemistry ${ }^{26}$. One remarkable observation was the absence of motion on as-prepared Au/Pt pumps. An activation step with oxygen plasma was needed to trigger the electrohydrodynamic process in $\mathrm{H}_{2} \mathrm{O}_{2}$. Such dramatic differences in pump performance are illustrated in Figure 5 which shows comparatively the positive tracer trajectories on samples with and without oxygen plasma treatment. Oxygen plasma removes resin contaminants from devices fabricated by electron-beam lithography. As a 
consequence, the catalyst becomes more active for triggering the chemomechanical actuation. Micropumps fabricated from stencils are free from chemicals and resins but still, the activating plasma treatment was necessary.

X-ray photoelectron spectroscopy (XPS) and electrochemical characterization based on Tafel measurements were performed to understand the treatment effects and to better assess the surface chemistry impact on the electrochemical behavior of the surfaces. Tafel measurements are very useful to determine the mixed potentials of platinum and gold, that is, the potential at which there is no net redox reaction and the current is zero ${ }^{30}$. Extracting the mixed potentials helps to predict the redox role of such metals when they are electrically connected in presence of redox species. The metal which exhibits a more positive mixed potential would act as cathode whereas the metal with a more negative mixed potential would act as anode ${ }^{26,30}$. The higher the difference between mixed potentials, the higher is the driving force for the catalytic actuation. Figure 5 shows the Tafel plots for $\mathrm{Au}$ and Pt electrodes in presence of $\mathrm{H}_{2} \mathrm{O}_{2}$ without and with plasma treatment. In absence of the cleaning treatment, the mixed potentials for Au and Pt are very similar. After the treatment, the mixed potential of Pt appears at a higher voltage than that of Au. These measurements confirmed the experimental results obtained with charged tracers: i) plasma treatment increases the driving force for the electrochemical reactions, boosting fluid motion and ii) the unexpected role of $\mathrm{Au}$ as anode and $\mathrm{Pt}$ as cathode in our micropumps.

XPS measurements revealed that the plasma treatment not only can remove remaining contaminants but also modify the Au/Pt surface chemistry by adding oxygen moieties. Figure 5 shows the XPS spectra of Au4f and Pt4f core levels for the untreated (e,g) and plasma treated (f,h) samples, respectively. The untreated samples show the characteristic spin-orbit splitting doublet corresponding to the metallic bonding. However, in the treated samples, additional 
shoulders at higher binding energies are observed arising from oxidized Au and Pt states. These results clearly show that pretreatment changes the surface chemistry of the metals which finally has an impact on the electrochemical reaction rates. Moreover, the presence of oxygen functionalities (oxides and hydroxides) on such metals can contribute to confer more negative zeta potential to the catalysts and hence promote the fluid motion. We know from numerical analysis that the fluid velocity increases with the zeta potential, being largely depressed for weakly charged surfaces ${ }^{27}$.

We believe that the surprising opposite redox role of $\mathrm{Au}$ and $\mathrm{Pt}$ in swimmers versus pumps roots in the high sensitivity of electrocatalysis on metal surface chemistry. The swimmer manufacturing undergoes a series of fabrication steps (metal electrodeposition in templates, removal of metallic sacrificial layers, removal of the template, etc.) which could contribute to having other adsorbates, altering the surface chemistry and hence the electrochemical response $\mathrm{e}^{20,30}$. 

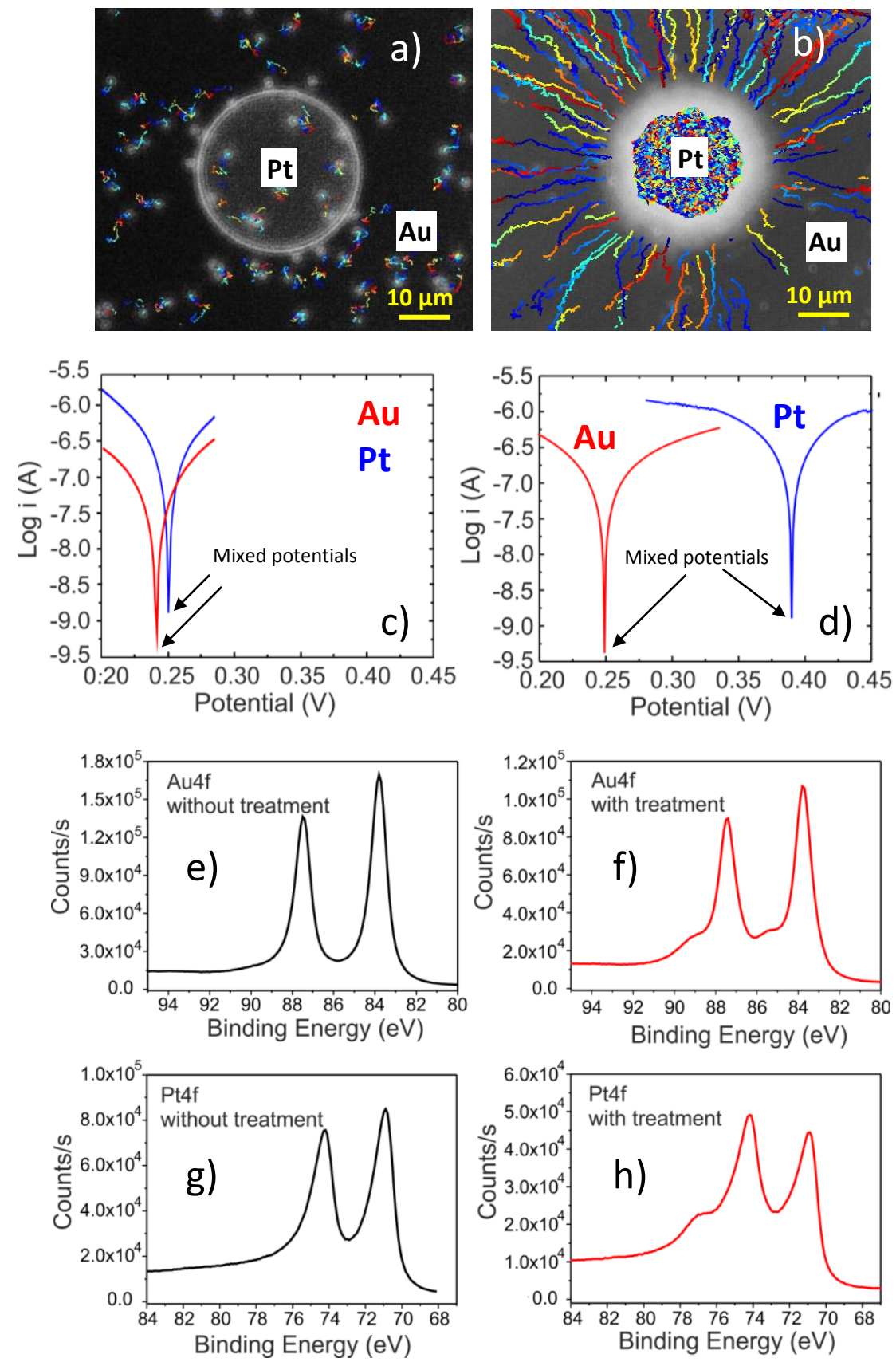

Figure 5. Optical images of $\mathrm{Au} / \mathrm{Pt}$ pumps interacting with positive particles. The images show the particle trajectories at the pumps without (a) and with plasma treatment (b). Tafel plots of Au and Pt before (c) and after plasma treatment (d). XPS Au4f and Pt4f spectra corresponding to 
surfaces without (e and g) and with plasma treatment ( $\mathrm{f}$ and h). Figures $5 \mathrm{~d}$-h reprinted with permission from ref. 26. Copyright 2013 by The American Physical Society.

\section{METAL/SEMICONDUCTOR PUMPS}

\subsection{Photochemical activation with visible light}

The use of light for on-demand motion control is very attractive given its fast switchable capabilities and wireless/remote propagation. In this section, we introduce a new example of how pumps can be used to probe photoactivated reactions for triggering fluid motion ${ }^{28}$. These studies are very useful to test the performance of different materials as a first step towards the development of optimized photochemical swimmers, which are more complex to characterize and manufacture. In this case, the micropump was made of $\mathrm{Pt}$ or $\mathrm{Au}$ discs patterned on silicon and could be controlled with visible light provided by the low electronic energy band-gap of silicon $^{28}$. Surprisingly the pump could be fueled just by water under visible illumination. However, an enhancement of the catalytic actuation was achieved when using more reactive

species such as $\mathrm{H}_{2} \mathrm{O}_{2}$. Again by applying the set of techniques described previously, we could extract the electrokinetic parameters and image the proton gradient which is the typical product of anodic reactions when using hydrogen-containing fuels. Figure 6a shows a proton gradient map in water using fluorescent $\mathrm{pH}$ indicators activated in the visible range. High proton concentration is observed on the silicon side, whereas proton depletion is observed on the metal side. Although the exact photochemical pathway is not well known, the protons production at silicon supports anodic reactions mediated by water at the semiconductor interface, whereas the proton decrease on the metal side is compatible with proton consumption due to cathodic reactions at the Pt interface ${ }^{28}$. By contrast, no proton gradient was generated when an insulating 
layer is placed between the metal and the semiconductor, as depicted in Figure 6b. This finding suggested that electron transfer between the semiconductor and the metal is important to make this pump operative.

The interaction of the pump with differently charged particles also supported the redox behavior of $\mathrm{Si}$ as anode and $\mathrm{Pt}$ as cathode. The electrohydrodynamic process was triggered just in presence of the optical microscope white light. Positive tracers moved toward the Pt disc and stuck on it, negative particles were repelled from the Pt disc, and quasi-neutral tracers moved to the disc and then drifted upwards. Again the proton gradient and the trajectories of charged particles indicated an electro-osmotic mechanism with a self-generated electric field and fluid flow pointing in the same direction from $\mathrm{Si}$ to the Pt disc. Interestingly the photogenerated electric field with water as fuel was found to be rather weak $(80 \mathrm{~V} / \mathrm{m}$, almost 4 times smaller than that in Au/Pt pumps). Despite the weak electric field, the fluid velocity was comparatively higher $(\simeq 9 \mu \mathrm{m} / \mathrm{s})$ than in the case of the Au/Pt device $(\simeq 6 \mu \mathrm{m} / \mathrm{s})$. Such striking behavior is a consequence of the more negative zeta potential of $\mathrm{Si}$ as compared to those of noble metals, which makes the electro-osmotic fluid motion sizable even though the electric field generated by the reaction is weak (eq. 4).

The photochemomechanical actuation of the silicon-based pump was largely amplified when adding $\mathrm{H}_{2} \mathrm{O}_{2}$. The tracer interaction with the pump followed the same trend as in the case of water but with a higher strength. Positive tracers approached the disc at high velocity (Fig. 6f) and the repulsion of negative tracers from the Pt disk became stronger. The self-generated electric field and fluid flow increased almost 9 and 5 times, respectively, as compared to those obtained in pure water ${ }^{28}$. 
The most remarkable aspect of these photochemically activated pumps was the tunability of the chemomechanical actuation with light intensity ${ }^{28}$. Light becomes a powerful tool to selectively control the electric field strength and fluid flow and hence the mass transport to specific locations. Figure $6 \mathrm{~g}$ shows the significant velocity decrease of positive tracers obtained by attenuating light intensity with a filter. Similarly, Fig. 6h shows the increase of the exclusion zone of negative tracers on $\mathrm{Si} / \mathrm{Pt}$ pumps achieved upon increasing light intensity. Photoactivation of the chemomechanical actuation can be framed as a photo-catalytic effect enhanced by the presence of the metal. Upon light absorption in silicon, electron-hole pairs are generated. The holes generated at the semiconductor become oxidizing agents for the fluid or for the silicon itself whereas the injection of electrons at the metal counterpart transforms it as a reducing agent. The transfer of generated electrons from a semiconductor to a metal ( $\mathrm{Au}$ or $\mathrm{Pt}$ ) through their common interface is a well-known process and has been exploited to increase the efficiency of photo-catalysis in different applications ${ }^{28}$.

Visible light-driven machines with the additional capability to work using just water hold relevant promise as switches for controlled mass transport in fluids but also for photoactivated driven swimmers operating with a more innocuous light source and fuel. In fact, recent studies have reported the development of visible light-driven $\mathrm{Si} / \mathrm{Au}$ swimmers in water whose speed was modulated with light intensity ${ }^{31}$. The authors postulated a photoactivated reaction with silicon oxidation and proton generation at the silicon side and proton reduction at the Au side ${ }^{31}$. This scenario correlates well with our proton gradient imaging and the redox role of Si as anode and the noble metal as cathode ${ }^{28}$. 

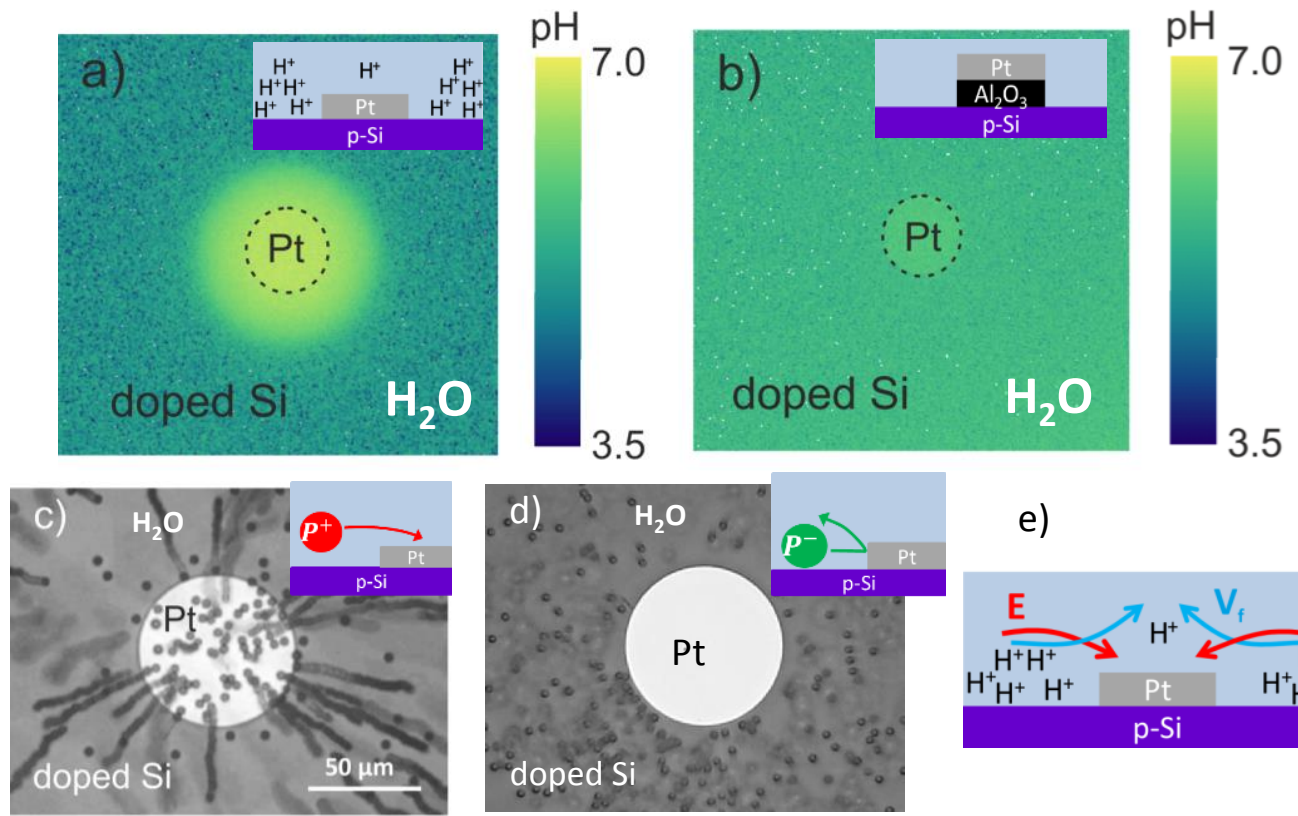

e)
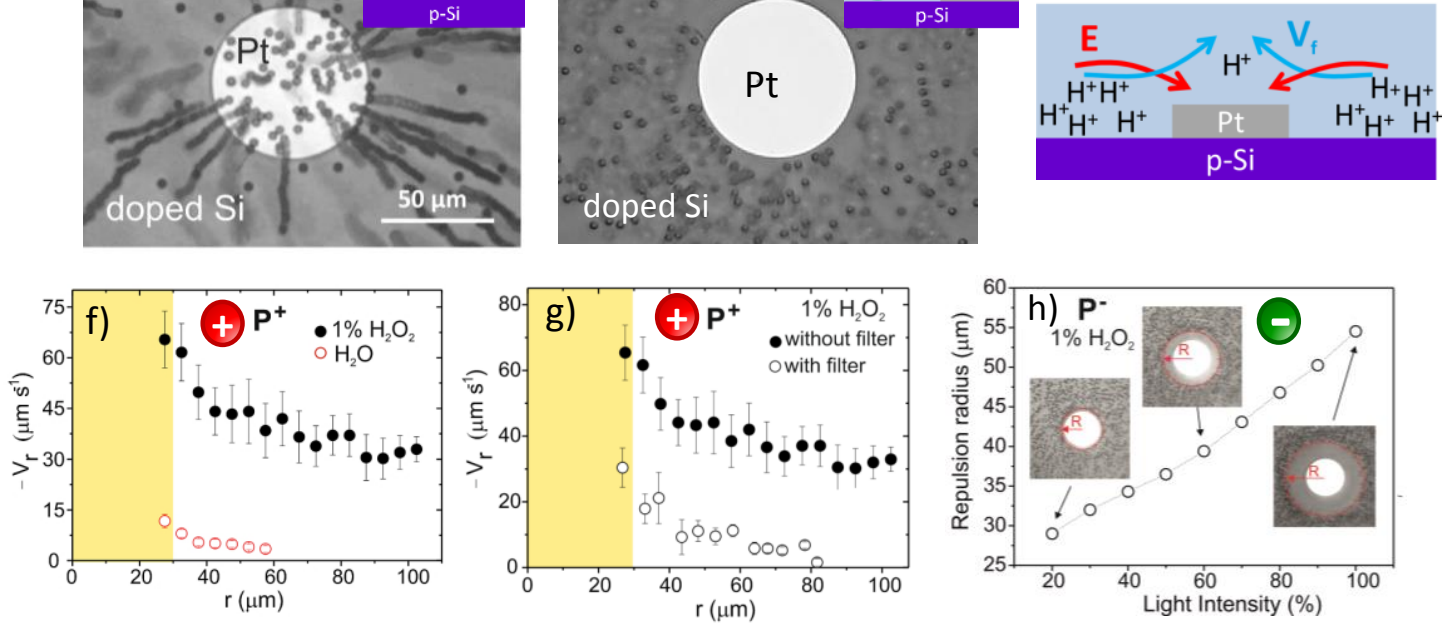

Figure 6. Mapping of the proton distribution on $\mathrm{Pt} / \mathrm{Si}$ pumps (a) and $\mathrm{Pt} / \mathrm{Al}_{2} \mathrm{O}_{3} / \mathrm{Si}$ in water (b). No proton gradient is generated at the pump when using an insulating layer between $\mathrm{Pt}$ and $\mathrm{Si}$. Motion of positive tracers towards Pt disc (c) and repulsion of negative tracers from Pt (d). e) Schematics depicting direction of fluid flow and electric field. f) Comparison of the radial velocity of positive tracers in $\mathrm{H}_{2} \mathrm{O}$ and $\mathrm{H}_{2} \mathrm{O}_{2}$. Light effect on positive (g) and negative (h) tracers. Figures 6 a-c and e-h reproduced with permission from ref. 28. Copyright 2015 by The American Chemical Society. 


\subsection{Competing mechanisms on light driven motion}

More than one chemomechanical mechanism can be operating on micro/nanomachines, and the prevalence of one of them over the others depends on the experimental and fabrication conditions. We studied comparatively pumps with different metal roughness to explore potential competing mechanisms ${ }^{29}$.

$\mathrm{Si} / \mathrm{Pt}$ micropumps with patterned Pt discs of the same size and thickness were fabricated using two different metal deposition methods: sputtering and electron-beam evaporation (used in the pumps from the previous section). Sputtered Pt discs exhibit larger roughness than that obtained through e-beam deposition ( $>10$ times). The impact of Pt roughness on the pump performance was elucidated by tracking the motion of charged tracers exposed to different light intensities. The interaction of positive and negative tracers with the rough-Pt/Si and smooth-Pt/Si devices was strikingly different as depicted in Figure 7 c-f. In the case of smooth Pt/Si pumps, positive tracers moved towards the Pt disc at high velocity and were trapped on it whereas negative tracers were repelled forming a particle-free halo from the Pt edge. However, in the case of the rough $\mathrm{Pt} / \mathrm{Si}$ pump, positive tracers moved toward $\mathrm{Pt}$ with much lower velocity and then drifted upward after crossing the Pt edge. More surprisingly, negative tracers moved towards the Pt disc and stuck on it. The quantification of the electrokinetic parameters revealed a remarkable decrease in the electric field strength (28 times lower) and in the fluid flow (6 times lower) in the rough Pt case as compared to the values obtained with the smoother pump. Moreover, for the rough $\mathrm{Pt}$ the motion of tracers did not show any significant variation with light intensity, in contrast to the smooth $\mathrm{Pt} / \mathrm{Si}$ device in which a remarkable decrease of the positive tracer velocity with light attenuation was observed. These findings suggested another mechanism taking over the photoactivated electro-osmotic one. 

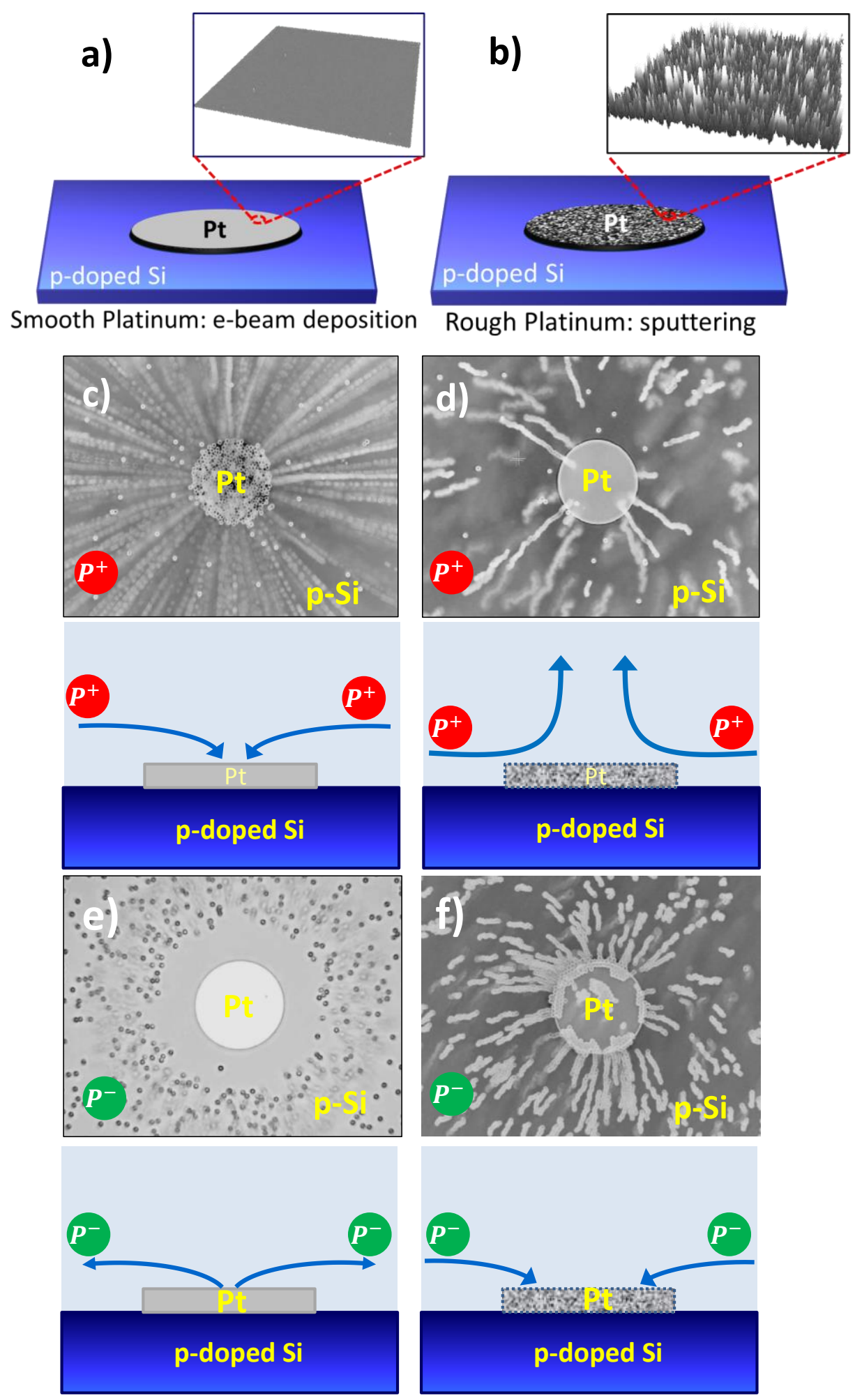

Figure 7. Schematics of smooth (a) and rough (b) Pt/Si pumps together with their respective $\mathrm{Pt}$ topographic AFM images taken at the same $\mathrm{z}$ scale. c-f) Interaction of positive and negative 
tracers with smooth and rough pumps. Schematic drawings of the particle trajectories are also included for each pump. Reprinted with permission from ref. 29. Copyright 2017 by The American Chemical Society.

To better study such competing mechanism, the photoactivated electro-osmotic process was suppressed by introducing a thick insulating layer $(1 \mu \mathrm{m})$ of $\mathrm{SiO}_{2}$ between $\mathrm{Si}$ and $\mathrm{Pt}$, thus blocking the electron transfer between the semiconductor and metal. Interestingly the motion of positive and negative tracers in the $\mathrm{Pt} / \mathrm{SiO}_{2}$ followed the same trend than in the case of the rough $\mathrm{Pt} / \mathrm{Si}$ pump, being also light insensitive. Evaluation of the motion of different tracers yielded a fluid flow pointing towards Pt and a small electric field pointing outwards, in the opposite direction to the one observed in the photoactivated electro-osmosis. The electric field strength was too weak to repel the positive tracers from the Pt surface. As a result, the positive tracer motion was dominated by the fluid flow which pointed inward to the Pt disc. It is important to highlight that such weak electric field cannot explain a chemomechanical actuation based on electro-osmosis since it should have triggered a fluid flow in the same direction of the electric field, given the negative zeta potential of the pump. Therefore, the fluid driving mechanism in the rough $\mathrm{Pt} / \mathrm{SiO}_{2}$ pumps correlates better with a diffusio-osmosis process induced by the direct decomposition of $\mathrm{H}_{2} \mathrm{O}_{2}$ at the rough Pt surface. As a result, the fluid moves towards the region where the reaction takes place, that is, the Pt disc where the solute concentration is higher. The origin of the small electric field is still an open question.

The findings with $\mathrm{Pt} / \mathrm{SiO}_{2}$ pump help to understand the degraded light-driven performance of rough Pt/Si pumps in view of two competing mechanisms, as illustrated in Fig. 8. One of them is 
the electro-osmotic process triggered by the photoactivated reaction which takes place in two separated redox reactions at $\mathrm{Si}$ and $\mathrm{Pt}$, generating an electric field and fluid flow in the same direction toward Pt. This mechanism would prevail on smooth $\mathrm{Pt} / \mathrm{Si}$ devices. The other mechanism seems to be neutral diffusio-osmosis, since the small electric field is not responsible for triggering fluid flow. Such mechanism could be rooted in the direct decomposition of $\mathrm{H}_{2} \mathrm{O}_{2}$ on Pt without Si mediation, provided by the excellent catalytic properties of Pt. The $\mathrm{H}_{2} \mathrm{O}_{2}$ decomposition at the Pt region would generate a fluid flow towards the Pt structure to compensate the higher solute concentration and higher osmotic pressure. This process is not light responsive and such chemical pathway would be more favored on rough $\mathrm{Pt} / \mathrm{Si}$ devices ${ }^{29}$. 


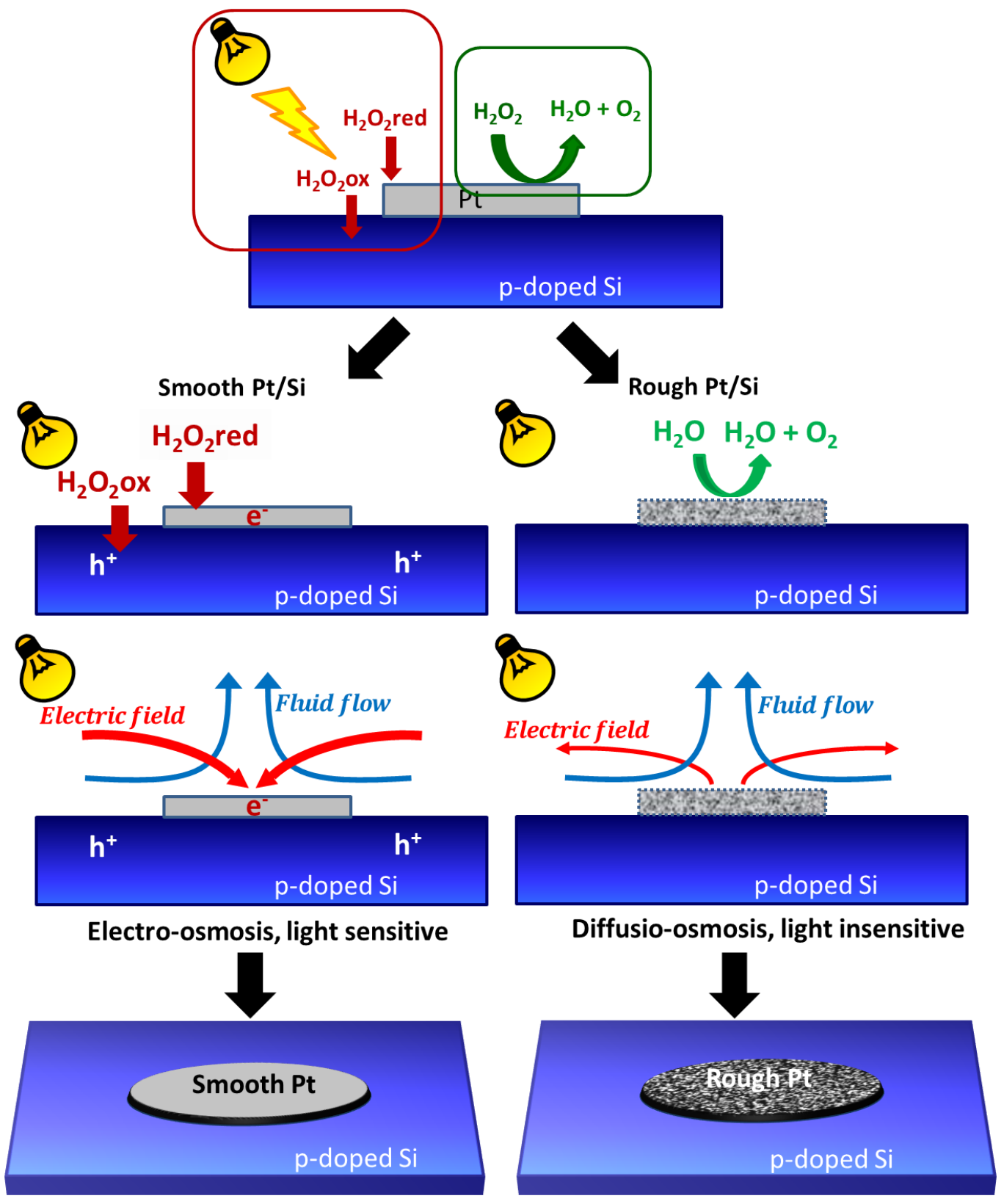

Figure 8. Schematics depicting the two competing mechanisms: the photoactivated electroosmosis which prevails on smooth $\mathrm{Pt} / \mathrm{Si}$ pumps and the light insensitive diffusio-osmosis which dominates on rough Pt/Si. Reprinted with permission from ref. 29. Copyright 2017 by The American Chemical Society. 
We have also found depression of the photoactivated mechanism when fabricating $\mathrm{Pt} / \mathrm{Si}$ swimmers with the Pt segment deposited with sputtering. Figure 9 shows a SEM image of Si/Pt swimmers together with their velocities when immersed in $1 \% \mathrm{H}_{2} \mathrm{O}_{2}$. The swimmer velocities did not show a clear trend with light intensity. The figure also compares the swimmer motion when a thick insulating layer $(100 \mathrm{~nm})$ of $\mathrm{SiO}_{2}$ is introduced between $\mathrm{Si}$ and $\mathrm{Pt}$ to completely block the electron transfer between them and hence the light driven half reactions taken place separately at Si and Pt segments. In this case the swimmers moved with slightly lower velocity and their motion was insensitive to light as expected for a diffusiophoretic process. In view of the previous results, the direct $\mathrm{H}_{2} \mathrm{O}_{2}$ decomposition on $\mathrm{Pt}$ is the dominating chemical pathway at rough $\mathrm{Pt} / \mathrm{Si}$ swimmers, inducing a chemomechanical actuation controlled more by a diffusiophoretic mechanism.
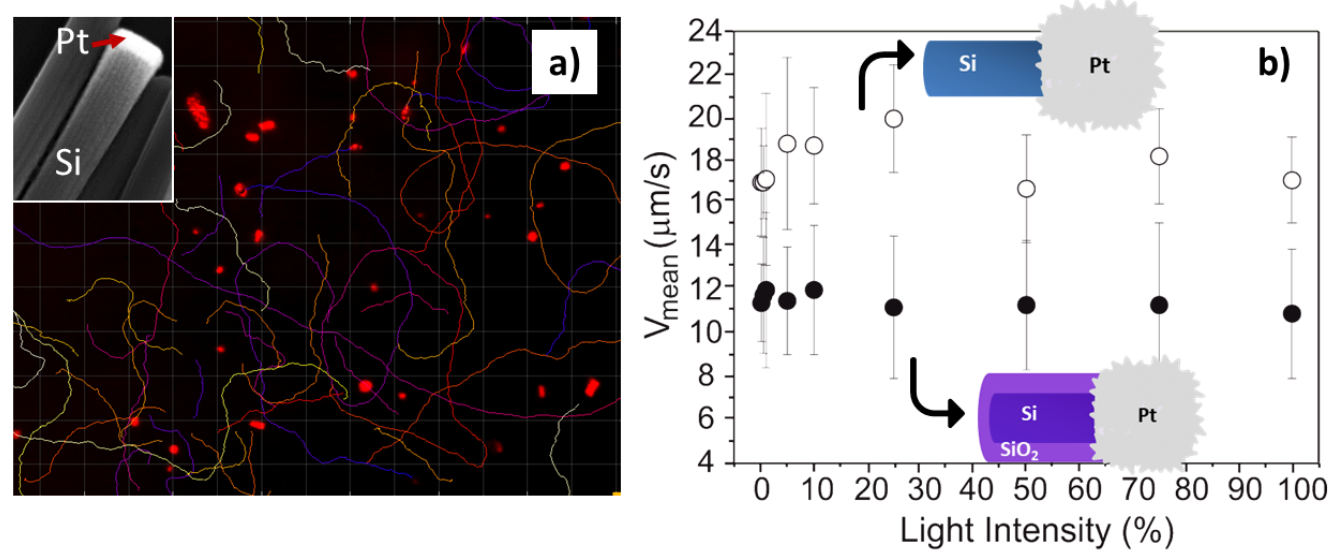

c)

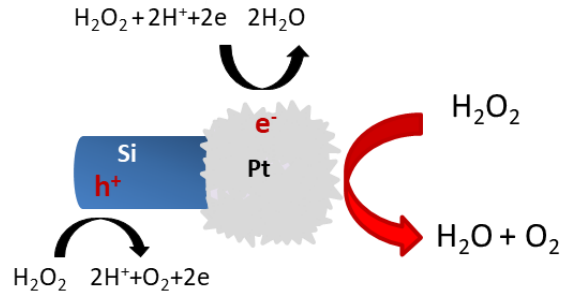

Figure 9. a) SEM image and trajectories of rough $\mathrm{Pt} / \mathrm{Si}$ swimmers. The silicon rods were fabricated in p-doped silicon wafers using colloidal nanolithography and metal (Au) assisted 
chemical etching. Next, a Pt cap $(20 \mathrm{~nm})$ was deposited on the Si rods by sputtering at grazing angle. The Si/Pt nanorods were finally treated with oxygen plasma and released to the fluid by sonication. b) Mean velocity of rough $\mathrm{Pt} / \mathrm{Si}$ and rough $\mathrm{Pt} / \mathrm{SiO}_{2}$ swimmers vs light intensity. c) Schematics of the competing chemical pathways: the photoactivated $\mathrm{H}_{2} \mathrm{O}_{2}$ decomposition taken place separately at $\mathrm{Si}$ and $\mathrm{Pt}$; and the light-insensitive decomposition of $\mathrm{H}_{2} \mathrm{O}_{2}$ taken place only at Pt.

\section{Conclusions}

Beyond their potential applications, micropumps, as immobilized motors, are ideal candidates for understanding the complex interrelation between electrochemical reactions and hydrodynamics. We have implemented a combined set of experimental and theoretical tools to systematically study their chemomechanical actuation and accomplish a better understanding, quantification, and identification of the key parameters controlling fluid motion. This has been exemplified by applying such methodology to bimetallic and light driven semiconductor/metal pumps. By mapping the proton gradient and quantifying the spatial distribution of key electrokinetic parameters such as the electric field and fluid flow, it has been possible to identify the motion mechanisms. We have shown that surface chemistry is crucial in setting the roles of anode/cathode at motors and hence on controlling the redox reaction direction. We have also identified two competing mechanisms on semiconductor/metal pumps: photoactivated electroosmosis and diffusio-osmosis, stemming from different chemical pathways in the fuel decomposition. We have found that surface roughness becomes crucial to dictate the prevailing chemical pathway and consequently boost or suppress one mechanism over the other. 
All these experimental and theoretical studies with pumps open new avenues for a better understanding and design of their swimmer counterparts. Novel fuels, materials and driving mechanisms can be tested providing useful clues for the engineering and optimization of phoretic systems.

\section{AUTHOR INFORMATION}

\section{Corresponding Author}

*E-mail: mariajose.esplandiu@icn2.cat.

Biographical Information

Maria J. Esplandiu received her PhD in the National University of Cordoba (Argentina) and was postdoctoral fellow at the universities of Dresden and Ulm (Germany), UCLA and CALTECH. She is currently a CSIC tenured scientist at the Catalan Institute of Nanoscience and Nanotechnology. Her research focuses on surface (electro)chemistry, surface nanoengineering and interfacial phenomena for applications in carbon based nanoelectronics, sensors and motors.

Kuan Zhang received his double master's degrees from Grenoble Institute of Technology (France) and University of Augsburg (Germany). He is currently a PhD candidate at the Catalan Institute of Nanoscience and Nanotechnology.

Jordi Fraxedas obtained his $\mathrm{PhD}$ from the University Stuttgart (Germany) and held a postdoctoral position at the European Synchrotron Radiation Facility (Grenoble, France) and a 
researcher position at the European Laboratory for Particle Physics (Geneva, Switzerland). He is currently Group Leader at the Catalan Institute of Nanoscience and Nanotechnology. His research activity is focused on interfacial phenomena and surface science.

Borja Sepulveda received his $\mathrm{PhD}$ degree in Physics from the Complutense University of Madrid in 2005 and was a postdoctoral fellow in Chalmers University of Technology (Göteborg, Sweden). He is currently a CSIC tenured scientist at the Catalan Institute of Nanoscience and Nanotechnology. His research lines include nanophotonics, magnetoplasmonics, and nanofabrication for biomedical and environmental applications.

David Reguera is Associate Professor at the University of Barcelona, where he obtained his $\mathrm{PhD}$ in Physics in 2001. He was a postdoctoral researcher at UCLA. His research lines focus on biophysics of viruses, nucleation, and non-equilibrium statistical mechanics.

\section{ACKNOWLEDGMENT}

This research was supported by the Spanish Ministry of Economy and Competitiveness, (MINECO) under Contract Nos. MAT2015-68307-P, FIS2015-67837 and M-ERA.NET Project PCIN2016-093. The ICN2 is funded by the CERCA program/ Generalitat de Catalunya. The ICN2 is supported by the Severo Ochoa program of MINECO (Grant SEV-2013-0295).

\section{REFERENCES}

(1) Paxton, W.F.; Kistler, K.C.; Olmeda, C.C.; Sen, A.; St Angelo, S.K.; Cao, Y.Y.; Mallouk, T.E.; Lammert, P.E.; Crespi, V.H. Catalytic nanomotors: autonomous movement of striped nanorods. J. Am. Chem. Soc. 2004, 126, 13424-13431. 
(2) Fournier-Bidoz, S.; Arsenault, A.C.; Manners, I.; Ozin, G.A. Synthethic self-propelled nanorotors. Chem. Commun. 2005, 441-443.

(3) Sengupta, S.; Ibele, M.E., Sen, A. Fantastic voyage: Designing self-powered nanorobots. Angew. Chem. Int. Ed. 2012, 51, 8434-8445.

(4) Wang. W.; Duan, W.; Ahmed, S.; Mallouk, T.E.; Sen, A. Small power: autonomous nanoand micromotors propelled by self-generated gradients. Nano Today 2013, 8, 532-554.

(5) Sanchez, S.; Soler, L.; Katuri, J. Chemically powered micro- and nanomotors. Angew. Chem. Int. Ed. 2015, 54, 1414-1444.

(6) Pumera, M. Electrochemically powered self-propelled electrophoretic nanosubmarines. Nanoscale 2010, 2, 1643-1649.

(7) Gao, W.; Wang, J. Synthetic micro/nanomotors in drug delivery. Nanoscale 2014, 6, 1048610494.

(8) Gao, W.; Esteban-Fernández de Avila, B; Zhang, L.; Wang, J. Targeting and isolation of cancer cells using micro/nanomotors. Adv. Drug Deliv. Rev. 2018, 125, 94-101.

(9) Vilela, D.; Cossío, U.; Hortelao, A.C.; Balderas-Xicohtencatl, R.; Hirscher, M.; Hahn, K.; Ma. X.; Sanchez, S.; Parmar J.; Martinez-Villacorta, A.M.; Gómez-Vallejo, V.; Llop, J.; Sanchez, S. Medical imaging for the tracking of micromotors. ACS Nano 2018, 12, 1220-1227.

(10) Li, J.; Esteban- Fernandez de Avila, B.; Gao, W.; Zhang, L.; Wang, J. Micro/Nanorobots for biomedicine: delivery, surgery, sensing and detoxification. Science Robotics 2017, 2, aam6431. 
(11) Soler, L.; Sanchez, S. Catalytic nanomotors for environmental monitoring and water remediation. Nanoscale 2014, 6, 7175-7182.

(12) Gao, W.; Wang, J. The environmental impact of micro/nanomachines. A review. ACS Nano 2014, 8, 3170-3180.

(13) Safdar, M.; Simmchen, J.; Jänis, J. Light-driven micro- and nanomotors for environmental remediation. Environ. Sci.:Nano 2017, 4, 1602-1616.

(14) Wang, W.; Duan, W.; Ahmed, S.; Sen, A.; Mallouk, T.E. From one to many: dynamic assembly and collective behavior of self-propelled colloidal motors. Acc. Chem. Res. 2015, 48, 1938-1946.

(15) Ashar-Farniya, A.; Esplandiu, M.J.; Bachtold, A. Sequential tasks performed by catalytic pumps for colloidal crystallization. Langmuir 2014, 30, 11841-11845.

(16) Zhou, C.; Zhang, H.; Li, Z.; Wang, W. Chemistry pumps: a review of chemically powered micropumps. Lab Chip, 2016, 16, 1797-1811.

(17)Wong, F.; Dey, K.; Sen, A. Synthethic micro/nanomotors and pumps: fabrication and applications. Annu. Rev. Mater. Res. 2016, 46, 407-432.

(18) Anderson, J.L. Colloid Transport by Interfacial Forces, Annu. Rev. Fluid Mech. 1989, 21, 61-99.

(19) Yadav, V.; Duan, W.; Butler, P.J.; Sen, A. Anatomy of nanoscale propulsion. Annu. Rev. Biophys. 2015, 44, 77-100. 
(20) Paxton, W.F.; Baker, Kline, T.R.; Wang, Y.; Mallouk, T.E.; Sen, A. Catalytically induced electrokinetics for motors and pumps. J. Am. Chem. Soc. 2006, 128, 14881-14888.

(21) Kline, R.T.; Iwata, J.; Lammert, P.E.; Mallouk, T.E.; Sen, A.; Velegol, D. Catalytically driven colloidal patterning and transport. J. Phys. Chem. B 2006, 110, 24513-24521.

(22) Moran, J.L.; Posner, J.D. Electrokinetic locomotion due to reaction-induced charge autoelectrophoresis. J. Fluid Mech. 2011, 680, 31-66.

(23) Moran, J.L.; Posner, J.D. Phoretic self-propulsion. Annu. Rev. Fluid Mech. 2017, 49, 511540.

(24) Brown, A.; Poon, W. Ionic Effects in Self-Propelled Pt-Coated Janus Swimmers, Soft Matter. 2014, 10, 4016-4027.

(25) Ebbens, S.; Gregory, D.A.; Dunderdale, G.; Howse, J.R.; Ibrahim Y.; Liverpool, T.B.; Golestanian, R. Electrokinetic Effects in Catalytic Pt-Insulator Janus Swimmers, Europhys. Lett. 2014, 106, 58003.

(26) Afshar-Farniya, A.; Esplandiu, M.J.; Reguera, D.; Bachtold, A. Imaging proton concentration and mapping the spatial distribution of the electric field of catalytic micropumps. Phys. Rev. Lett. 2013, 111, 168301.

(27) Esplandiu, M.J.; Afshar Farniya, A.; Reguera, D. Key parameters controlling the performance of catalytic motors. J. Chem. Phys. 2016, 144, 124702. 
(28) Esplandiu, M.J.; Afshar Farniya, A.; Bachtold, A. Silicon-based chemical motors: an efficient pump for triggering and guiding fluid motion using visible light. ACS Nano 2015, 9, $11234-11240$.

(29) Zhang, K.; Fraxedas, J.; Sepulveda, J.; Esplandiu, M.J. Photochemically activated motors:

from electrokinetic to diffusion motion control. ACS Appl. Mater. Interfaces 2017, 9, 4494844953.

(30) Wang Y.; Hernandez R.M.; Bartlett D.J.; Bingham J.M.; Kline T.R.; Sen A.; Mallouk T.E. Bipolar electrochemical mechanism for the propulsion of catalytic nanomotors in hydrogen peroxide solutions. Langmuir 2006, 5, 10451-10456.

(31) Zhou, D.; Li, Y.C.; Xu, P.; Ren, L.; Zhang, G.; Mallouk, T.E.; Li, L. Visible-light driven SiAu micromotors in water and organic solvents. Nanoscale 2017, 32, 11434-11438. 

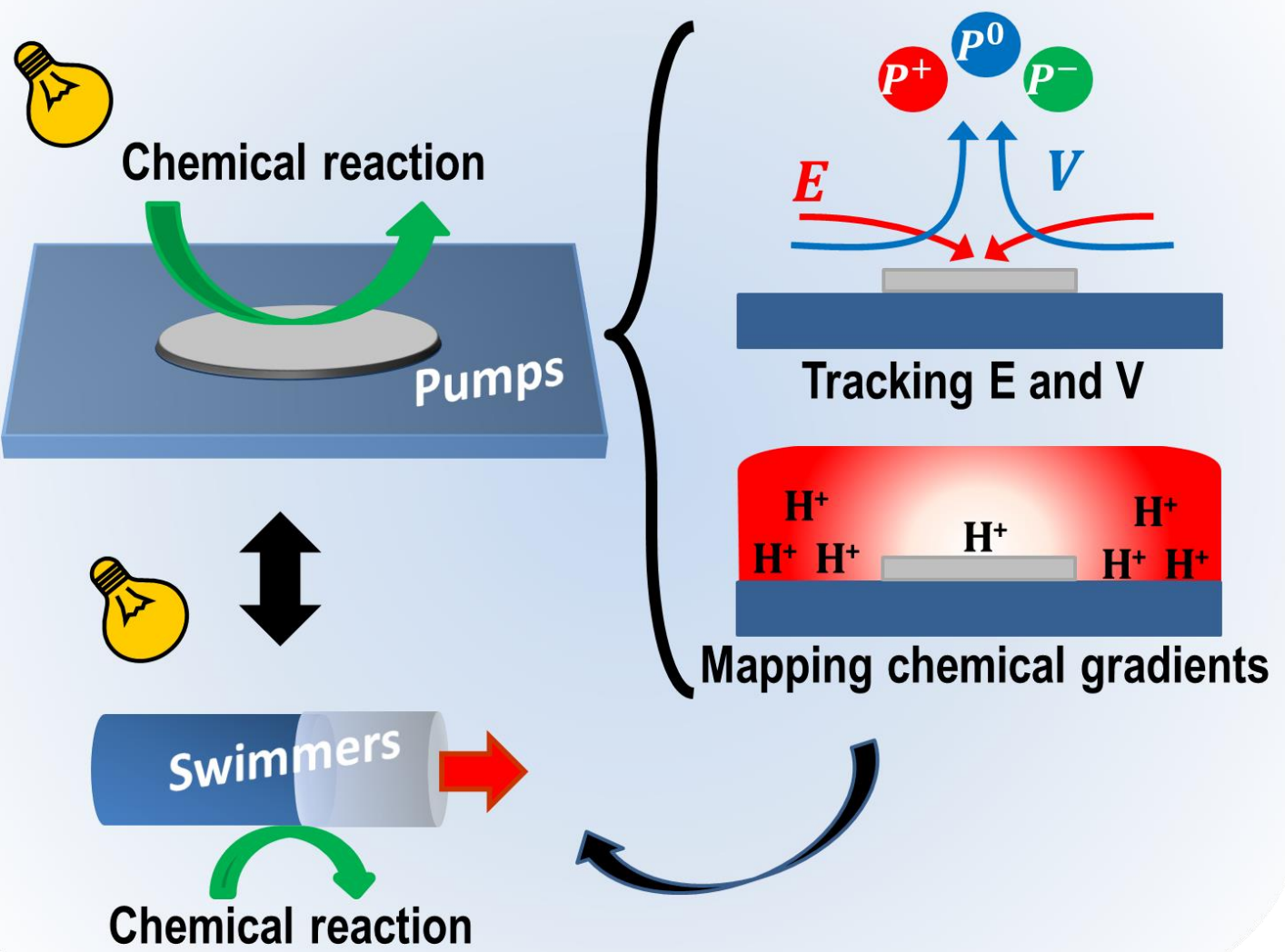